\title{
Comparative effectiveness of coronary screening in heart valve surgery: Computed tomography versus conventional coronary angiography
}

\author{
Wonjae Lee, MD, ${ }^{\mathrm{a}}$ Joon Bum Kim, MD, PhD, ${ }^{\mathrm{b}}$ Dong Hyun Yang, MD, PhD, ${ }^{\mathrm{c}}$ Cherry Kim, MD, PhD, \\ Jihoon Kim, MD, PhD, ${ }^{\mathrm{b}}$ Min Ho Ju, MD, ${ }^{\mathrm{b}}$ Ho Jin Kim, MD, ${ }^{\mathrm{b}}$ Joon-Won Kang, MD, PhD, ${ }^{\mathrm{c}}$ \\ Sung-Ho Jung, MD, PhD, ${ }^{\mathrm{b}}$ Young-Hak Kim, MD, PhD, ${ }^{\mathrm{d}}$ Suk Jung Choo, MD, PhD, ${ }^{\mathrm{b}}$ \\ Cheol Whan Lee, MD, PhD, ${ }^{\mathrm{d}}$ Cheol Hyun Chung, MD, PhD, ${ }^{\mathrm{b}}$ Jae Won Lee, MD, PhD, ${ }^{\mathrm{b}}$ and \\ Tae-Hwan Lim, MD, $\mathrm{PhD}^{\mathrm{c}}$
}

\section{ABSTRACT}

Background: Although conventional coronary angiography (CAG) is considered the gold standard for coronary artery disease (CAD) screening in the setting of heart valve surgery, coronary artery computed tomography angiography (CCTA) has emerged as an alternative modality. This study was conducted to evaluate the clinical outcomes of CCTA compared with conventional CAG for CAD screening in patients undergoing heart valve surgery.

Methods: A total of 3150 consecutive patients aged $>40$ years or with coronary risk factors undergoing elective valve operations between 2001 and 2015 were evaluated. Of these, 1402 patients underwent CCTA (CT group) and 1748 patients underwent conventional CAG (CAG group) for CAD screening.

Results: The 30-day mortality rates were similar in the 2 groups $(2.1 \%$ in the CT group vs $1.7 \%$ in the CAG group; $P=.463)$; however, the incidence of low cardiac output syndrome was higher in the CT group $(2.3 \%$ vs $1.0 \% ; P=.008)$. The final rate of detection of significant $\operatorname{CAD}(\geq 50 \%$ stenosis) $(4.9 \%$ vs $9.7 \%$; $P<.001)$ and proportion of receiving coronary bypass grafting (CABG) $(2.9 \%$ vs $4.3 \% ; P=.041$ ) were lower in the CT group. After adjustment by propensity score matching (563 pairs), the main findings of our crude analyses did not change, with lower rates of CAD detection (odds ratio [OR], 0.56; 95\% confidence interval $[\mathrm{CI}], 0.36-0.85)$ and $\mathrm{CABG}(\mathrm{OR}, 0.47 ; 95 \% \mathrm{CI}, 0.26-0.81)$, a similar risk of early mortality (OR, $1.51 ; 95 \% \mathrm{CI}, 0.54-4.52)$, but a higher risk of low cardiac output syndrome (OR, 3.30; 95\% CI, 1.16-11.78) in the CT group compared with the CAG group.

Conclusions: The detection of significant CAD and identification of candidates for CABG were inferior with CCTA compared with conventional CAG in patients scheduled for elective heart valve operations. ( $\mathrm{J}$ Thorac Cardiovasc Surg 2018;155:1423-31)

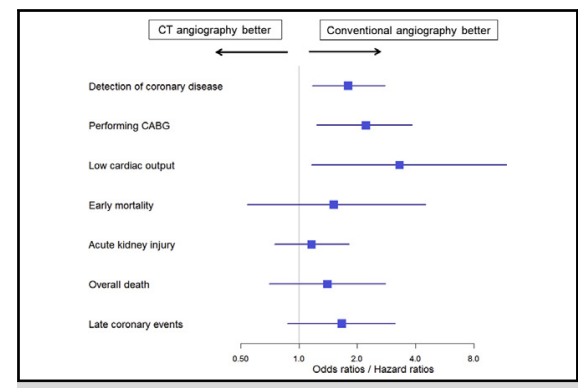

Comparative outcomes between CCTA and conventional CAG in heart valve surgery.

\section{Central Message}

Computed tomography angiography may be inferior to conventional coronary angiography in detecting coronary disease and in identifying candidates for coronary artery bypass grafting among patients scheduled for heart valve operations.

\section{Perspective}

Coronary artery computed tomography angiography (CCTA) may be inferior to conventional coronary angiography (CAG) in detecting coronary disease and in identifying candidates for coronary artery bypass grafting among patients scheduled for heart valve operations. Further prospective researches are needed to validate the feasibility of CCTA compared with conventional CAG.

See Editorial Commentary page 1432.

\footnotetext{
From the a Division of Cardiology, Department of Internal Medicine, College of Medicine, Seoul National University and Cardiovascular Center, Seoul National University Bundang Hospital, Seongnam-si, Gyeonggi-do, Korea; ${ }^{b}$ Departments of Thoracic and Cardiovascular Surgery and ${ }^{\mathrm{c}}$ Radiology, and ${ }^{\mathrm{d}}$ Division of Cardiology, University of Ulsan College of Medicine, Asan Medical Center, Seoul, Korea; and ${ }^{\mathrm{e}}$ Department of Radiology, Ansan Hospital, Korea University College of Medicine, Ansan-si, Gyeonggi, Korea.

Drs Kim and Yang contributed equally to this work.

This study was supported by a grant from the Asan Institute for Life Sciences, Asan Medical Center, Seoul, Korea.

Read at the 97th Annual Meeting of The American Association for Thoracic Surgery, Boston, Massachusetts, April 29-May 3, 2017.
}

Received for publication July 11, 2017; revisions received Oct 2, 2017; accepted for publication Oct 27, 2017; available ahead of print Dec 12, 2017.

Address for reprints: Joon Bum Kim, MD, PhD, Department of Thoracic and Cardiovascular Surgery, Asan Medical Center, University of Ulsan College of Medicine, 388-1 Pungnap-dong Songpa-gu, Seoul 138-736, South Korea (E-mail: jbkim1975@amc.seoul.kr). or Dong Hyun Yang, MD, PhD, Department of Radiology, Asan Medical Center, University of Ulsan College of Medicine, 388-1 Pungnap-dong Songpa-gu, Seoul 138-736, South Korea (E-mail: donghyun. yang@gmail.com). $0022-5223 / \$ 36.00$

Copyright (C) 2017 by The American Association for Thoracic Surgery https://doi.org/10.1016/j.jtcvs.2017.10.128 


$$
\begin{aligned}
& \text { Abbreviations and Acronyms } \\
& \begin{aligned}
\text { AKI } & =\text { acute kidney injury } \\
\text { CABG } & =\text { coronary artery bypass grafting } \\
\text { CAD } & =\text { coronary artery disease } \\
\text { CAG } & =\text { coronary angiography } \\
\text { CCTA } & =\text { coronary computed tomography } \\
& \text { angiography } \\
\text { CI } & =\text { confidence interval } \\
\text { CT } & =\text { computed tomography } \\
\text { HR } & =\text { hazard ratio } \\
\text { LCO } & =\text { low cardiac output syndrome } \\
\text { MACE } & =\text { major adverse cardiac event } \\
\text { OR } & =\text { odds ratio } \\
\text { PS } & =\text { propensity score }
\end{aligned}
\end{aligned}
$$

(U) $\begin{aligned} & \text { Scanning this QR code will take } \\ & \text { you to supplemental tables and } \\ & \text { figures for this article. }\end{aligned}$

Current practice guidelines recommend screening for coronary artery disease (CAD) before cardiac surgery in patients aged $>40$ years or with coronary risk factors, to reduce the risk of complications related to perioperative myocardial ischemia. ${ }^{1-3}$ For this, conventional coronary angiography (CAG) has been considered the gold standard method for detecting obstructive CAD and has commonly been used for CAD evaluation before surgery. However, the invasive nature of CAG has called for an alternative modality that is noninvasive while still as effective. Coronary computed tomography angiography (CCTA) has been validated for its accuracy and efficacy in detecting CAD in many clinical settings. ${ }^{4}$ With the introduction of 320 -slice and 640-slice CT, the accuracy of CCTA has improved further, with less radiation and with better performance even in the presence of tachyarrhythmias, such as atrial fibrillation. ${ }^{5,6}$ In perioperative $\mathrm{CAD}$ screening for patients undergoing noncardiac surgery, CCTA is generally considered useful for excluding $\mathrm{CAD}$ in patients at low risk for atherosclerosis, and recently has been encouraged as an alternative to invasive $\mathrm{CAG}^{7,8}$

Currently, CCTA is suggested as an alternative approach for preoperative $\mathrm{CAD}$ evaluation in patients with low or intermediate pretest likelihood of CAD undergoing elective heart valve operations (class IIa recommendation). ${ }^{3}$ Based on several recent studies have shown excellent diagnostic accuracy of CCTA in the preoperative assessment for elective valve surgery to rule out the presence of significant
$\mathrm{CAD},{ }^{9-11}$ some have suggested expanding the role of CCTA as a gatekeeper before invasive CAG in patients undergoing valve surgery. ${ }^{12}$ Given relatively small sample sizes of these recent studies and the questionable reproducibility of the results of such studies, there have been calls for further studies performed in real-world clinical settings with reasonable cohort sizes to validate the feasibility of CCTA compared with conventional CAG. Thus, in the present study, we sought to compare CCTA and invasive CAG as CAD screening modalities in patients undergoing elective heart valve surgery in terms of perioperative and long-term clinical outcomes.

\section{METHODS}

\section{Study Subjects and Outcome Measures}

In a review of the institutional prospective cardiac surgical database in Asan Medical Center, Seoul, Korea, we identified 6104 consecutive patients age $>40$ years or with a coronary risk factor (ie, diabetes, hypertension, dyslipidemia, or severe [obesity body mass index $\geq 30 \mathrm{~kg} / \mathrm{m}^{2}$ ]) undergoing heart valve surgery between January 2001 and December 2015. After excluding patients with emergent surgery, infective endocarditis, or preexisting coronary disease, 3212 patients were judged to meet our enrollment criteria. Of these, 3150 patients who underwent preoperative coronary imaging studies by either conventional CAG $(n=1748$; CAG group) or CCTA ( $n=1402$; CT group) composed the study cohort.

The choice of imaging modality was affected by the pretest probability of coronary disease, with CCTA preferred in low-risk patients, but was finally at the surgeon's discretion. In addition, attributed to its less invasive nature combined with the advent of the more accurate CCTA, there was a strong trend toward increasing use of CCTA over CAG in recent years. The majority of patients included in this study were referred to our institution, a large-volume tertiary-referral center, from other hospitals for surgical treatment under the diagnosis of severe cardiac valvulopathy. The study protocol was approved by the Institutional Review Board of the Asan Medical Center. The requirement for informed consent from individual patients was waived from the Board due to the retrospective study design.

The primary objective of this study was to compare the detection rate of significant CAD ( $>50 \%$ stenosis) confirmed by CAG, and the rate of concomitant performance of coronary artery bypass grafting (CABG), between the CAG and CT groups. For further measures, early complications, including mortality; acute kidney injury (AKI); low cardiac output syndrome, defined by hemodynamic collapse requiring mechanical support (extracorporeal life support or intra-aortic balloon pump); and neurologic injuries occurring within 30 days after an index surgery, were also evaluated. AKI was defined as a $>2$-fold increase in the serum creatinine, or a $50 \%$ decrease in glomerular filtration rate based on RIFLE (risk, injury, failure, loss, and end-stage kidney disease) criteria. ${ }^{13}$

Late follow-up events potentially relevant to $\mathrm{CAD}$, including all-cause mortality, requirement for coronary revascularization, and myocardial infarction, were also reviewed and compared as a composite endpoint representing a major adverse cardiac event (MACE). For data collection, additional retrospective chart reviews were conducted to obtain detailed information on perioperative variables and follow-up outcomes.

\section{Coronary Imaging}

Systemic, thorough reviews of CCTA and CAG findings were conducted by expert radiologists and interventional cardiologists, respectively. These imaging data were summarized in 9 parameters, as proposed in the CONFIRM registry. ${ }^{14}$ The degree of stenosis was classified as minimal $(<30 \%)$, mild $(30 \%-49 \%)$, moderate $(50 \%-69 \%)$, or severe $(\geq 70 \%)$. In general, the presence of moderate-to-severe coronary stenosis, calcification 
TABLE 1. Baseline characteristics before and after propensity score matching

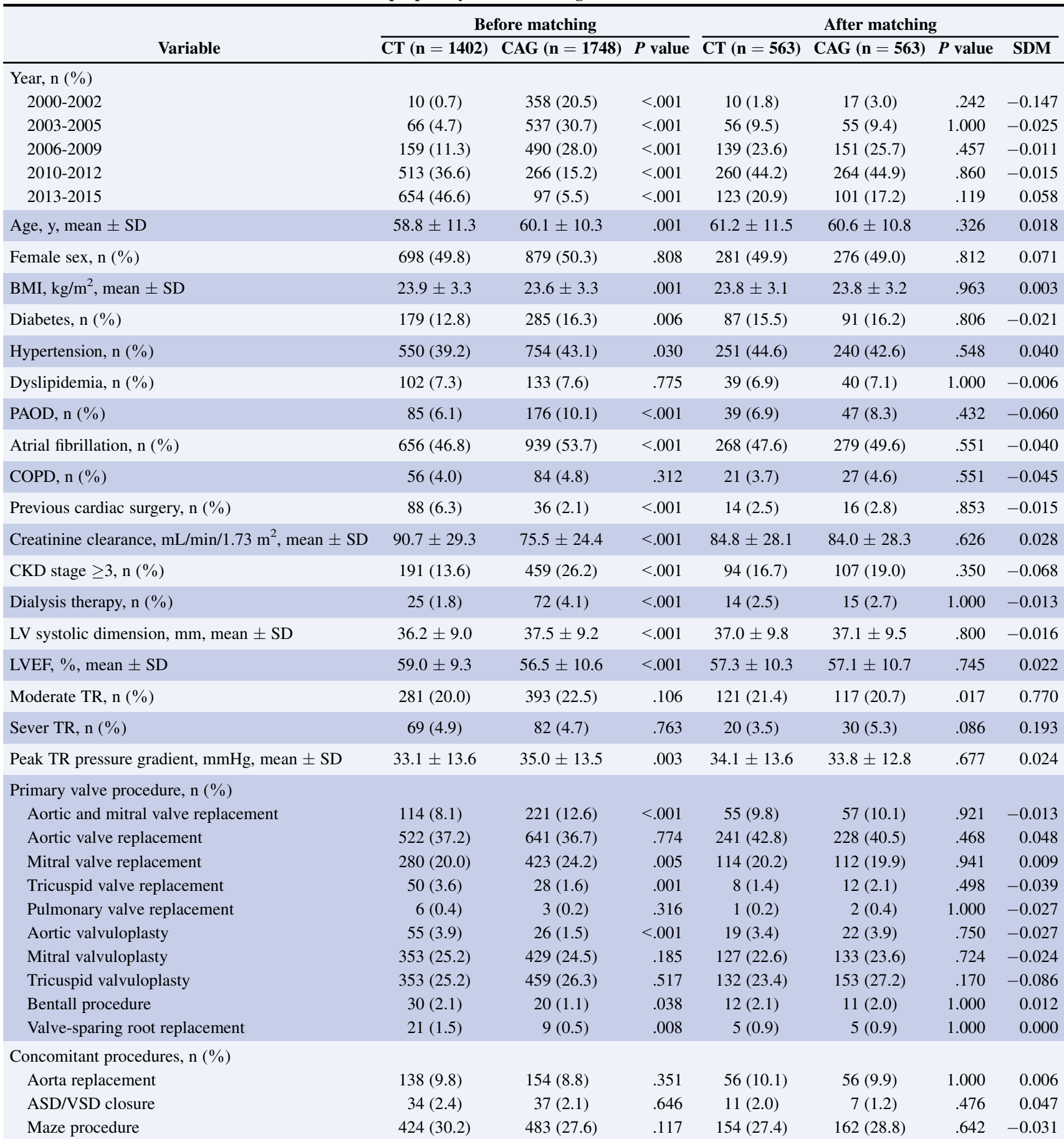

$C T$, Computed tomography; $C A G$, coronary angiography; $S D M$, standardized difference of the mean; $S D$, standard deviation; $B M I$, body mass index; $P A O D$, peripheral artery occlusive disease; $C O P D$, chronic obstructive pulmonary disease; $C K D$, chronic kidney disease; $L V$, left ventricle; $L V E F$, left ventricle ejection fraction; $T R$, tricuspid regurgitation; $A S D$, atrial septal defect; $V S D$, ventricular septal defect.

of the coronary arteries, or artifact that hindered adequate evaluation of stenosis on CCTA were considered indications for further evaluation by conventional CAG.

CCTA was performed with single-source 16-slice CT (Sensation 16; Siemens Medical Solutions, Forchheim, Germany) before January 2007, and with a first- or second-generation dual-source CT scanner (Somatom
Definition or Somatom Definition Flash; Siemens Medical Solutions) thereafter. Patients with no contraindication to $\beta$-adrenergic blocking agents and with an initial heart rates $>65 \mathrm{bpm}$ received an oral dose of $2.5 \mathrm{mg}$ of bisoprolol 1 hour before undergoing CCTA. CCTA scanning was conducted in conformance with established guidelines and technical parameters. ${ }^{15,16} \mathrm{CT}$ parameters are summarized in Table E1. 


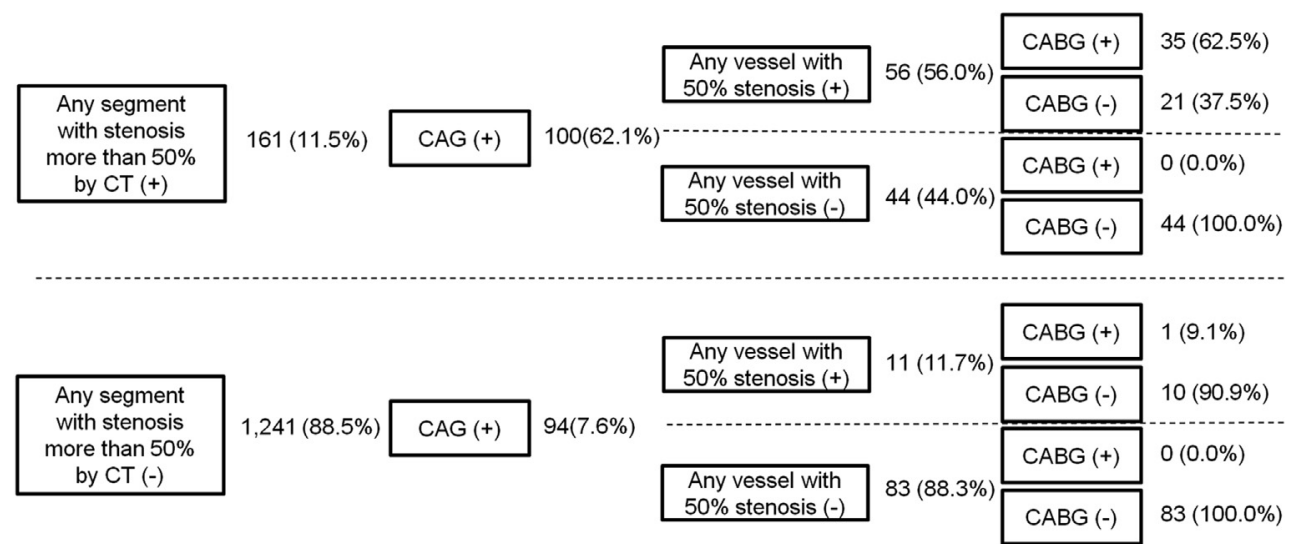

FIGURE 1. Clinical pathway of patients who initially underwent computed tomography coronary angiography for screening $(\mathrm{n}=1420)$. $C T$, computed tomography; $C A G$, coronary angiography; $C A B G$, coronary artery bypass grafting.

\section{Statistics}

All statistical analyses were performed using R version 3.3.1 (R Foundation for Statistical Computing, Vienna, Austria). Categorical variables, presented as frequencies and percentages, were compared using the $\chi^{2}$ test or Fisher's exact test. Continuous variables are expressed as mean \pm standard deviation or as median with range (or interquartile range [IQR]), and the assumption of normality for these variables was assessed with the Shapiro-Wilk test. Based on the test result for each variable, Student's unpaired $t$ test or Mann-Whitney $U$ test was used for the appropriate comparison. The Kaplan-Meier method was used to analyze time-related events, and log-rank tests were used to compare intergroup differences in such event rates.

To reduce the influence of selection bias between the 2 coronary imaging modalities, we adjusted the differences in the patient's baseline covariates using propensity score (PS) analysis. ${ }^{17}$ The PS, which represents the probability of undergoing CCTA instead of CAG in each patient based on baseline covariates, was estimated using a multiple logistic regression model including all baseline covariates listed in Table 1, as well as the year of surgery (in quintiles). The calibration and discrimination capacities of the PS model were assessed using Hosmer-Lemeshow statistics and C statistics, respectively. Then the PS-matched pairs were created by 1:1 matching between the CT and CAG groups on the logit of the PS using nearest-neighbor matching, allowing a maximal caliper of width $\leq 0.10$ of the standard deviation of the logit of the PS. The balance of the baseline covariates after PS matching was assessed by examining the standardized difference of the mean, with a value $<0.1$ considered well balanced for each covariate. ${ }^{18}$ Comparisons in the PS-matched pairs were performed using the Wilcoxon rank-sum test for continuous variables and McNemar's test for binary data.

Conditional logistic regression analysis was performed for evaluating the early outcomes, and Cox proportional hazard models were used to evaluate the late time-related outcomes (death and MACEs) in the PS-matched cohort. For further validation of the results from PS matching, covariate adjustment with PS was performed for the entire cohort. PS was incorporated into the logistic regression or Cox-Hazard model as a covariate and type of imaging modality (CT vs CAG) to calculate the propensityadjusted odds ratio (OR) or hazard ratio (HR). We examined $\log (-\log [$ survival]) curves and partial (Schoenfeld) residuals to test the proportional hazards assumption in the Cox-Hazard models, and found no relevant violations in the models for any of the time-related outcomes.

For sensitivity analysis, we examined the difference in the incidence of late stroke within the PS-matched cohort. Because we would not expect a difference in the rate of late stroke depending on the initial coronary imaging modality, a significant difference in this late outcome may indicate a violation of our statistical assumption that the probability of undergoing one imaging modality (CT) over the other (CAG) depended on the observed baseline covariates alone. ${ }^{18}$ All reported $P$ values were 2-tailed, and $P \leq .05$ was considered statistically significant.

\section{RESULTS \\ Baseline Characteristics}

Baseline characteristics of 1748 patients in CAG group and 1402 patients in CT group are reported in Table 1. Patients in the CAG group tended to have higher clinical risk profiles compared with those in the CT group. Patients in CAG group were older, had a higher body mass index, and higher rates of diabetes, hypertension, peripheral artery disease, chronic kidney disease, and atrial fibrillation. Left ventricular ejection fraction was lower and peak tricuspid regurgitation pressure gradient was higher in the CAG group. The distribution of primary valve procedures and procedure types were significantly different between the 2 groups (Table 1).

The year of surgery had a significant influence on the choice of coronary imaging modality, with a strong tendency toward a preference of CCTA over conventional CAG over time throughout the entire study period (Table 1).

\section{Clinical Pathway in the CT Group}

A total of 194 patients underwent CCTA initially, followed by CAG for further evaluation (Figure 1). Among 1402 patients in the CT group, 161 patients $(11.5 \%)$ had a segment with $>50 \%$ stenosis detected by CCTA. In 100 of the 194 patients $(62.1 \%)$, CCTA had detected significant stenosis. Of these, $56(56.0 \%)$ were confirmed to have significant stenosis $(\geq 50 \%)$ by CAG, and $35(62.5 \%)$ underwent concomitant $\mathrm{CABG}$ surgery. For patients with severe coronary stenosis, the diagnostic pathway with the use of higher cutoff value of $\geq 70 \%$ stenosis detected on the initial CCTA is shown in Figure E1. Ninety-four patients underwent CAG even though CCTA did not show significant 
TABLE 2. CT parameters of CT then CAG and CT-only groups

\begin{tabular}{|c|c|c|c|}
\hline CT parameter & $\begin{array}{l}\text { CT, then CAG } \\
\begin{array}{c}(\mathbf{n}=194), \\
\text { n }(\%)\end{array}\end{array}$ & $\begin{array}{c}\text { CT-only } \\
(\mathbf{n}=\mathbf{1 2 0 8}), \\
\mathbf{n}(\%)\end{array}$ & $P$ value \\
\hline $\begin{array}{l}\text { Segments with any plaque or } \\
\text { stenosis }>50 \%\end{array}$ & & & $<.001$ \\
\hline 1 & $28(14.4)$ & $166(13.7)$ & \\
\hline 2 & $14(7.2)$ & $111(9.2)$ & \\
\hline$\geq 3$ & $111(57.2)$ & $222(18.4)$ & \\
\hline $\begin{array}{l}\text { Segments with }>50 \% \\
\text { stenosis }\end{array}$ & & & $<.001$ \\
\hline 1 & 37 (19.1) & $38(3.1)$ & \\
\hline 2 & $23(11.9)$ & $16(1.3)$ & \\
\hline$\geq 3$ & $38(19.6)$ & $7(0.6)$ & \\
\hline $\begin{array}{l}\text { Segments with }>70 \% \\
\text { stenosis }\end{array}$ & & & $<.001$ \\
\hline 1 & $33(17)$ & $17(1.4)$ & \\
\hline 2 & $15(7.7)$ & $6(0.5)$ & \\
\hline$\geq 3$ & $16(8.2)$ & $3(0.2)$ & \\
\hline $\begin{array}{l}\text { Proximal segments with } \\
\quad>50 \% \text { stenosis }\end{array}$ & & & $<.001$ \\
\hline 1 & $43(22.2)$ & $25(2.1)$ & \\
\hline 2 & $16(8.2)$ & $2(0.2)$ & \\
\hline$\geq 3$ & $8(4.1)$ & $0(0)$ & \\
\hline $\begin{array}{l}\text { Segments with noncalcified } \\
\text { plaque }\end{array}$ & & & $<.001$ \\
\hline 1 & $37(19.1)$ & $98(8.1)$ & \\
\hline 2 & $7(3.6)$ & $27(2.2)$ & \\
\hline$\geq 3$ & $6(3.1)$ & $16(1.3)$ & \\
\hline Segments with mixed plaque & & & $<.001$ \\
\hline 1 & $18(9.3)$ & $69(5.7)$ & \\
\hline 2 & $19(9.8)$ & $28(2.3)$ & \\
\hline$\geq 3$ & $19(9.8)$ & $17(1.4)$ & \\
\hline $\begin{array}{l}\text { Segments with calcified } \\
\text { plaque }\end{array}$ & & & $<.001$ \\
\hline 1 & $24(12.4)$ & 155 (12.8) & \\
\hline 2 & $19(9.8)$ & $87(7.2)$ & \\
\hline$\geq 3$ & $70(36.1)$ & $159(13.2)$ & \\
\hline
\end{tabular}

$C T$, Computed tomography; $C A G$, coronary angiography.

stenosis. Of these, $11(11.7 \%)$ had significant stenosis confirmed by CAG, and $1(9.1 \%)$ underwent subsequent CABG surgery. In addition, there were 3 cases of unplanned CABG surgery due to intraoperative coronary complications despite negative $\mathrm{CAG}$ results.

All CT parameters related to coronary artery atherosclerosis differed significantly between the patients who underwent CCTA only and those who were further evaluated by CAG (Table 2). The numbers of segments with any type of plaque, any stenosis, or proximal lesions were significantly higher in the patients further evaluated by CAG. When the diagnostic accuracy of CCTA was evaluated in the 194 patients who underwent both CCTA and CAG, assuming CAG as the gold standard, the sensitivity and specificity for detecting CAD of $>50 \%$ stenosis were $83.6 \%$ (56 of 67 ) and $65.4 \%$ (83 of 127 ), respectively, with positive and negative predictive values of $56.0 \%$ (56 of 100 ) and $88.3 \%$ (83 of 94), respectively.

\section{Perioperative Outcomes}

The final confirmation rates of significant $(>50 \%)$ coronary stenosis by CAG were $9.7 \%$ in the CAG group and $4.9 \%$ in the CT group $(P<.001)$ (Table 3$)$. For severe $(\geq 70 \%)$ stenosis, the final detection of CAD was still poorer in the CT group $(2.9 \%$ vs $4.9 \% ; P=.008)$. In addition, concomitant $\mathrm{CABG}$ surgery was more frequent in the CAG group $(4.3 \%$ vs $2.9 \% ; P=.041)$ (Table E2). After the index surgery, incidence of low cardiac output syndrome (LCOS) was significantly higher in the CT group $(2.3 \%$ vs $1.0 \% ; P=.008$ ) (Table E3), whereas there were no significant between-group differences in the rate of 30-day mortality $(1.6 \%$ vs $1.3 \%, P=.450)$ (Table E4), 30-day stroke $(0.7 \%$ vs $0.6 \% ; P=.620)$, or AKI $(6.7 \%$ vs $6.3 \% ; P=.683)$, including the need for new renal replacement therapy $(5.4 \%$ vs $4.9 \% ; P=.566)$.

Subgroup analysis of the CT group revealed that compared with those undergoing CCTA only without subsequent CAG $(n=1208)$, patients undergoing CAG after initial assessment by CCTA $(\mathrm{n}=194)$ had a significantly higher incidence of postoperative AKI $(11.4 \%$ [22 of 194 ] vs $5.4 \%$ [ 65 of 1208]; $P=.002$ ), as well as a higher rate of new dialysis therapy ( $9.3 \%$ [18 of 194$]$ vs $4.2 \%$ [51 of 1208]; $P=.003)$.

\section{Late Outcomes}

Follow-up was completed in 2620 of the 3150 patients $(83.2 \%)$. The median duration of follow-up was 41.3 months (IQR, 13.8-81.2 months) on the entire cohort, and was significantly longer in the CAG group compared with the CT group (65.8 months [IQR, 26.3-103.3 months] vs 22.6 months [IQR, 7.8-47.1 months]; $P<.001$ ). During follow-up, 138 cases of MACE, along with 118 deaths, 16 cases of myocardial infarction, and 13 cases of coronary revascularization therapy, occurred, with no significant between-group differences in rates (Table 3 and Figure 2).

\section{Outcome Adjustments by PS Matching}

The PS model showed a Hosmer-Lemeshow goodnessof-fit $P$ value of 0.117 and a c-statistic of 0.902 , indicating reasonable calibration and discrimination capacity. PS matching yielded 563 pairs of patients (Figure E2) with well-balanced status in all measurable baseline covariates (Table 1). The comparative clinical outcomes after PS matching were essentially similar to those before matching. Compared with patients in the CAG group, fewer patients in the CT group were diagnosed with significant CAD (OR, $0.56 ; 95 \%$ confidence interval $[\mathrm{CI}] 0.36-0.85 ; P=.007$ ), and fewer underwent concomitant CABG (OR, 0.47; 95\% 
TABLE 3. Unadjusted and adjusted outcomes

\begin{tabular}{|c|c|c|c|c|c|c|c|c|c|c|c|}
\hline \multirow[b]{2}{*}{ Variable } & \multicolumn{3}{|c|}{ Overall cohort } & \multicolumn{5}{|c|}{ Propensity score matching } & \multicolumn{3}{|c|}{ Propensity score adjustment } \\
\hline & $\begin{array}{c}\text { CT } \\
(\mathrm{n}=1402), \\
\text { n }(\%)\end{array}$ & $\begin{array}{c}\text { CAG } \\
\left(\begin{array}{c}n=1748), \\
n(\%)\end{array}\right.\end{array}$ & $\begin{array}{c}P \\
\text { value }\end{array}$ & $\begin{array}{c}\text { CT } \\
(\mathbf{n}=563), \\
n(\%)\end{array}$ & $\begin{array}{c}\text { CAG } \\
(\mathrm{n}=\mathbf{5 6 3}), \\
\mathrm{n}(\%)\end{array}$ & OR/HR & $95 \% \mathrm{CI}$ & $P$ value & OR/HR & $95 \% \mathrm{CI}$ & $\begin{array}{c}P \\
\text { value }\end{array}$ \\
\hline \multicolumn{12}{|l|}{ Early outcomes } \\
\hline Confirmation of CAD* & $68(4.9)$ & $169(9.7)$ & $<.001$ & $37(6.6)$ & $63(11.2)$ & 0.56 & $0.36-0.85$ & .007 & 0.55 & $0.37-0.81$ & .003 \\
\hline Implementation of CABG & $40(2.9)$ & $75(4.3)$ & .041 & $19(3.4)$ & $39(6.9)$ & 0.47 & $0.26-0.81$ & .008 & 0.47 & $0.28-0.80$ & .005 \\
\hline $\begin{array}{l}\text { Low cardiac output } \\
\text { syndrome }\end{array}$ & $32(2.3)$ & $18(1.0)$ & .008 & $13(2.3)$ & $4(0.7)$ & 3.30 & $1.16-11.78$ & .038 & 2.82 & $1.26-6.32$ & .012 \\
\hline Mortality within $30 \mathrm{~d}$ & $23(1.6)$ & $23(1.3)$ & .450 & $9(1.6)$ & $6(1.1)$ & 1.51 & $0.54-4.52$ & .439 & 1.85 & $0.81-4.19$ & .143 \\
\hline Stroke within $30 \mathrm{~d}$ & $10(0.7)$ & $10(0.6)$ & .620 & $5(0.9)$ & $5(0.9)$ & 1.00 & $0.27-3.61$ & $>.99$ & 1.26 & $0.37-4.37$ & .720 \\
\hline Acute kidney injury & $88(6.3)$ & $117(6.7)$ & .638 & $46(8.2)$ & $43(7.6)$ & 1.16 & $0.75-1.81$ & .501 & 1.06 & $0.71-1.59$ & .782 \\
\hline Renal replacement therapy & $69(4.9)$ & $94(5.4)$ & .566 & $36(6.4)$ & $33(5.9)$ & 1.10 & $0.67-1.81$ & .706 & 1.15 & $0.73-1.81$ & .540 \\
\hline \multicolumn{12}{|l|}{ Late outcomes } \\
\hline Death & $43(1.22) \dagger$ & $75(0.75) \dagger$ & .856 & $19(0.95) \dagger$ & $15(0.70) \dagger$ & 1.40 & $0.70-2.79$ & .343 & 1.68 & $1.00-2.83$ & .049 \\
\hline $\begin{array}{l}\text { Major adverse cardiac } \\
\text { event }\end{array}$ & $50(1.42) \dagger$ & $88(0.88) \dagger$ & .693 & $24(1.20) \dagger$ & $16(0.75) \dagger$ & 1.66 & $0.87-3.17$ & .124 & 1.73 & $1.07-2.80$ & .025 \\
\hline Stroke & $42(1.19) \dagger$ & $103(1.03) \dagger$ & 720 & $20(1.00) \dagger$ & $28(1.30) \dagger$ & 0.89 & $0.49-1.61$ & 692 & 0.77 & $0.47-1.27$ & .305 \\
\hline
\end{tabular}

$C T$, Computed tomography; $C A G$, coronary angiography; $O R$, odds ratio; $H R$, hazard ratio; $C I$, confidence interval; $C A D$, coronary artery disease; $C A B G$, coronary artery bypass grafting. *Significant coronary artery stenosis defined as $\geq 50 \%$ luminal narrowing confirmed by coronary artery angiography. $\dagger$ Percent per patient-year. Low cardiac output syndrome is defined as requirement for mechanical hemodynamic support including intra-aortic balloon pumping, and extracorporeal membrane oxygenation. Major adverse cardiac event is a composite of mortality, coronary revascularization, and nonfatal myocardial infarction.

CI, 0.26-0.81; $P=.008$ ) (Table 3), but the risk of LCOS was higher in the CT group (OR, 3.30; 95\% CI, 1.16-11.78; $P=.038)$. Follow-up durations in the matched cohort were not significantly different between the 2 groups (median, 42.2 months [IQR, 17.5-65.0 months] in the CAG group vs 40.1 months [IQR, 15.7-60.7 months] in the CT group; $P=.086$ ) Other comparative outcomes, including overall mortality and the incidence of MACE during follow-up, were not different between the 2 groups (Figure 3). Finally, the incidence of late stroke in the matched cohort $(n=48)$, which served as a landmark for sensitivity analysis, was not significantly different between the 2 groups (HR, 0.89; 95\% CI, 0.49-1.61; $P=.692$ ).

Within the CT group, additional PS matching analyses were conducted between the CT- only subgroup $(n=1208)$ and the CT then CAG subgroup $(\mathrm{n}=194)$ to evaluate the impact of subsequent CAG on the risk of postoperative AKI in patients undergoing initial CCTA. PS matching yielded well-balanced 146 patient pairs, which showed increased risks of AKI (OR, 2.41; 95\% CI, 1.13-5.50; $P=.028)$ and the need for renal replacement therapy (OR, $3.07 ; 95 \%$ CI, $1.23-8.73 ; P=.022)$ with the addition of CAG.
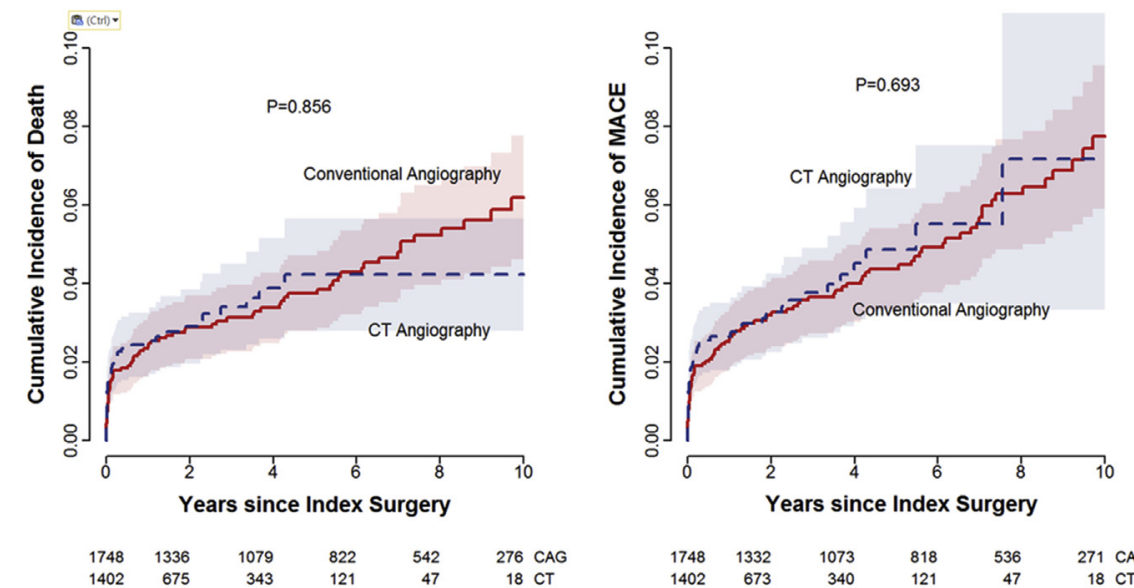

A

$\begin{array}{rrrrrr}1748 & 1336 & 1079 & 822 & 542 & 276 \text { CAG } \\ 1402 & 675 & 343 & 121 & 47 & 18 \text { CT }\end{array}$

\section{B}

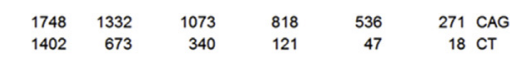

FIGURE 2. Unadjusted Kaplan-Meier plots for cumulative incidences of death (A) and major adverse cardiac event (MACE) (B) in the overall cohort. $C T$, Computed tomography; $C A G$, coronary angiography; $M A C E$, major adverse cardiac event. 


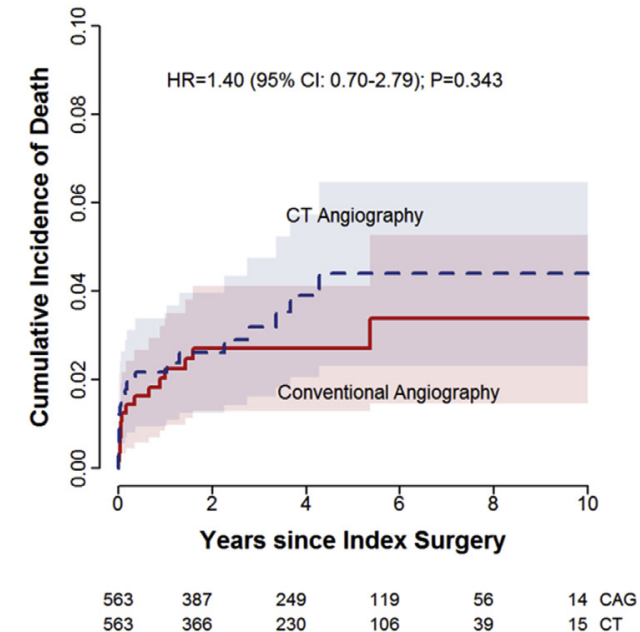

A

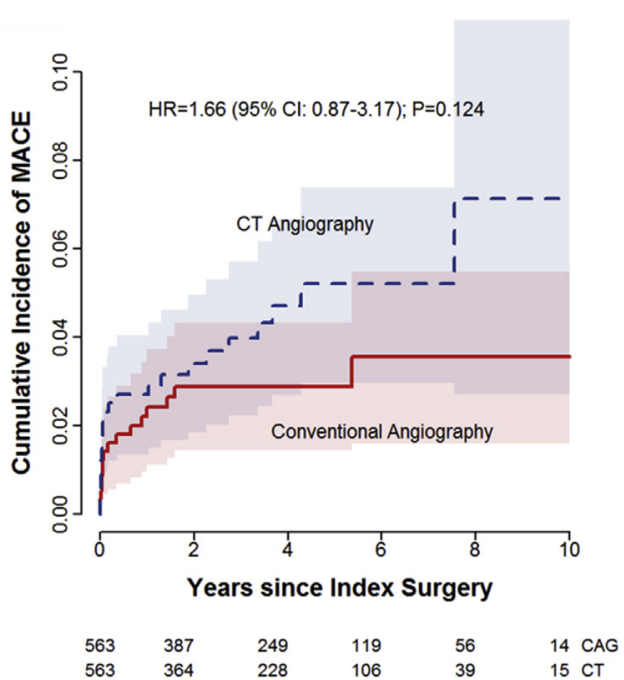

B

FIGURE 3. Adjusted Kaplan-Meier plots for cumulative incidences of death (A) and major adverse cardiac event (MACE) (B) in the propensity scorematched cohort. $H R$, Hazard ratio; $C I$, confidence interval; $C T$, computed tomography; MACE, major adverse cardiac event.

\section{DISCUSSION}

Our study results show no significant differences in mortality and MACE over the short term or long term when CCTA was initially used instead of CAG for screening CAD in patients undergoing valvular heart surgery. However, compared with CAG, CCTA identified fewer cases of significant $\mathrm{CAD}$, which may have contributed to the lower rate of concomitant $\mathrm{CABG}$ surgery in the $\mathrm{CT}$ group. In addition, this lower rate of concomitant $\mathrm{CABG}$ might have been associated with the higher incidence of LCOS in the CT group, raising questions about CCTA as an initial screening modality for CAD before surgery on a routine basis. We found no significant difference between the CT and CAG groups in the risk of AKI postsurgery; however, a subgroup analysis revealed an elevated risk of postoperative AKI in patients who underwent subsequent CAG following CCTA.

CCTA is widely used to evaluate CAD risk and to help identify candidates for concomitant $\mathrm{CABG}$ in patients undergoing valve surgery. CCTA is less invasive than CAG, and its accuracy is improving with the evolution of multidetector CT machines. Our center has increasingly used CCTA as an initial screening modality for CAD in recent years (Table 1). The current guideline suggests that CCTA can be an option to exclude CAD in patients considered at low or intermediate pretest risk of CAD. ${ }^{3}$ The level of evidence supporting this recommendation is fairly low, howev$\mathrm{er}$, and most previous studies have focused on the diagnostic accuracy of CCTA rather than on clinical outcomes of patients screened by CCTA. In particular, no study has validated whether CCTA can replace invasive CAG in this setting by comparing perioperative clinical outcomes with a large-sized cohort. Our present study is based on a large number of patients $(\mathrm{n}=3150)$ and their clinical outcomes, and thus may provide insight into clinical decision pathways for CABG and actual prognosis in the real world.

A recent noteworthy meta-analysis of studies evaluating the diagnostic accuracy of CCTA for preoperative CAD evaluation in patients with valvular heart disease included 17 studies analyzing 1107 patients and 12,851 coronary segments, which may provide robust power to suggest the potential for CCTA as a screening modality. ${ }^{19}$ Results included a pooled sensitivity of $93 \%$, specificity of $89 \%$, a negative likelihood ratio of 0.07 , and a positive likelihood ratio of 8.44. Although the authors concluded that CAG is not necessary when after a negative CCTA for CAD, their analysis was not explicit in terms of the heterogeneity of the results, or of population characteristics among the included studies; for instance, reported sensitivities ranged from $67 \%$ to $100 \%$, and specificities ranged from $80 \%$ to $100 \%$. In our study, per patient data showed a comparable sensitivity of $84 \%$, with a lower specificity of $65 \%$.

In our $\mathrm{CT}$ group, the confirmation rate of $\mathrm{CAD}$ was lower, at $6.6 \%$. It might be argued that there were some patients who had significant stenosis detected by CCTA but were not evaluated by CAG, which might have contributed to the lower rate of CAD detection in the CT group. Given the sensitivity of CCTA in our study, however, the CAD detection rate still might have been relatively low even had we assumed that all patients with 50\% stenosis detected by CCTA were evaluated by CAG. In addition, screening for $\mathrm{CAD}$ is done not only to evaluate perioperative risk, but also to identify patients in need of concomitant CABG. In general, concomitant coronary revascularization on CAD of $\geq 50 \%$ stenosis during valve surgery is considered reasonable according to the current practice guidelines (class IIa). ${ }^{3}$ In our present cohort, it seems evident that the 
surgeons could not identify as many candidates for CABG when patients were initially screened with CCTA. In this regard, substituting CAG with CCTA for CAD screening in patients undergoing valve surgery may be associated with suboptimal coronary revascularization, which may further increase the risk of serious perioperative myocardial injuries, the rate of which was higher in our CT group compared with our CAG group.

Our findings show that the patients in CT group were more susceptible to LCOS compared with those in CAG group $(2.3 \%$ vs $1.0 \% ; P=.008)$. Because the development of LCOS can be affected by a multitude of factors, the suboptimal detection of significant CAD by CCTA might not be the sole contributor to the increased incidence of LCOS in this large-sized heterogenous cohort with differing systolic function, cardiopulmonary bypass strategies, and procedural complexities. Owing to the very limited diagnostic and therapeutic approaches for patients with LCOS in the setting of mechanical circulatory support, we could not accurately assess the causality between remnant coronary lesions and occurrence of LCOS; nevertheless, given that LCOS was attributed mostly to perioperative myocardial infarction, the incidence of which was higher in the CT group (Table E3), the increased risk of LCOS in this group may be viewed as a reflection of suboptimal coronary revascularization by significant coronary lesions missed on CCTA.

Contrast-induced nephropathy is a not uncommon complication associated with further adverse outcomes in the short and long term. A meta-analysis with 138,603 patients confirmed that contrast-induced AKI after CAG is associated with an increased risk of mortality and cardiovascular events. ${ }^{20}$ Although definitions for the diagnosis of contrast- induced nephropathy vary, its reported incidence after CAG ranges from $3.3 \%$ to $16.5 \%{ }^{21} \mathrm{~A}$ recent meta-analysis of 42 studies (including $18,790 \mathrm{pa}-$ tients) of contrast-induced nephropathy following CT reported a pooled incidence of $4.96 \%{ }^{22}$ Another study reported $3.5 \%$ incidence of persistent renal dysfunction after CCTA. ${ }^{15}$ However, data are scarce on comparisons of the incidence of AKI between CAG and CCTA used as a screening tool in the setting of cardiac surgery. We analyzed postoperative AKI, which may be associated in part with the contrast media used for screening tests. We found no significant difference in the overall incidence of postoperative AKI between the CAG and CT groups; however, the increased risk of AKI in patients who underwent both CT and CAG for screening suggests that routine use of CCTA for screening will give rise to a substantial number of additional CAGs, which carries an increased risk of contrast-induced nephropathy. In these regard, selecting high-risk patients who would be potentially evaluated by CAG is important to avoid unnecessary screening by CCTA.
Regarding long-term clinical outcomes, the risks of mortality and MACEs were higher in the CT group compared with the CAG group in the matched cohorts (HR, 1.4 and 1.66), but these differences did not reach significance, perhaps because of the limited sample size after matching. However, in the PS-adjustment model (Table 3), the HRs were similar to those in the PS-matched analyses (1.68 for death, 1.73 for MACE), but the $P$ values were significant ( $P=.049$ and .025 , respectively), likely because the PSadjustment model retains all of the patients, preserving the sample power. Large-scale prospective data are needed to further verify the impact of preoperative imaging modality (CCTA vs CAG) on long-term mortality and incidence of MACE.

\section{Study Limitations}

Our study is purely observational, and a considerable number of baseline characteristics were significantly different between the 2 groups. The possibility exists that patients with a higher pretest probability of CAD were evaluated by CAG initially. In addition, there was a clear trend toward an increasing use of CCTA as a screening tool in recent years due to our center's policy. Although we sought to perform meticulous adjustments by PS matching as well as sensitivity analysis to overcome these potential biases, the inherent limitations of selection bias in observational studies might not have been fully addressed.

In addition, some patients $(\mathrm{n}=61)$ did not undergo further evaluation by CAG even if significant CAD was detected by CCTA, and some patients $(\mathrm{n}=94)$ underwent CAG even though no significant stenosis was detected by CCTA (Figure 2). This might be attributable to a practice pattern in real clinical settings without predefined strict regulations; however, the latter patient group $(\mathrm{n}=94)$ may provide valuable insight into the negative predictive value $(88.3 \%)$ of CCTA. Finally, even though numerous measurable baseline variables were included for PS analyses, patient symptoms were not accounted for in statistical adjustments.

\section{CONCLUSIONS}

This is the first large-scale study to compare CCTA and CAG as a screening tool in terms of perioperative and long-term outcomes in patients undergoing valvular heart surgery. Although mortality and MACE rates were not significantly different between the 2 groups, the strategy of using CCTA as an initial screening modality was associated with a lower detection rate of significant CAD, less concomitant CABG, and a higher incidence of LCOS. The incidence of postoperative AKI was higher in patients in whom both CCTA and CAG were performed, although no difference was observed between the CAG and CT groups. 


\section{Conflict of Interest Statement}

Authors have nothing to disclose with regard to commercial support.

\section{References}

1. Bonow RO, Carabello BA, Chatterjee K, de Leon AC Jr, Faxon DP, Freed MD, et al. ACC/AHA 2006 guidelines for the management of patients with valvular heart disease: a report of the American College of Cardiology/American Heart Association Task Force on Practice Guidelines (writing Committee to Revise the 1998 guidelines for the management of patients with valvular heart disease) developed in collaboration with the Society of Cardiovascular Anesthesiologists endorsed by the Society for Cardiovascular Angiography and Interventions and the Society of Thoracic Surgeons. J Am Coll Cardiol. 2006;48:e1-148.

2. Narang R, Chadha DS, Goel K, Mishra S, Bajaj N, Sharma S, et al. Screening coronary angiography prior to surgery in rheumatic valvular heart disease: a study of 2,188 patients. J Heart Valve Dis. 2009;18:455-60.

3. Nishimura RA, Otto CM, Bonow RO, Carabello BA, Erwin JP III, Guyton RA, et al. 2014 AHA/ACC guideline for the management of patients with valvular heart disease: a report of the American College of Cardiology/American Heart Association Task Force on Practice Guidelines. J Am Coll Cardiol. 2014;63: e57-185.

4. Budoff MJ, Dowe D, Jollis JG, Gitter M, Sutherland J, Halamert E, et al. Diagnostic performance of 64-multidetector row coronary computed tomographic angiography for evaluation of coronary artery stenosis in individuals without known coronary artery disease: results from the prospective multicenter ACCURACY (Assessment by Coronary Computed Tomographic Angiography of Individuals Undergoing Invasive Coronary Angiography) trial. J Am Coll Cardiol. 2008;52:1724-32.

5. Utsunomiya D, Oda S, Yuki H, Yamamuro M, Tsujita K, Funama Y, et al. Evaluation of appropriateness of second-generation 320-row computed tomography for coronary artery disease. Springerplus. 2015;4:109.

6. Lei Z, Fu Q, Shi H, Xu H, Han P, Yu J. The diagnostic evaluation of 640 slice computed tomography angiography in the diagnosis of coronary artery stenosis. Digit Med. 2015;1:67-71.

7. Kristensen SD, Knuuti J, Saraste A, Anker S, Bøtker HE, Hert SD, et al. 2014 ESC/ESA guidelines on non-cardiac surgery: cardiovascular assessment and management: the Joint Task Force on non-cardiac surgery: cardiovascular assessment and management of the European Society of Cardiology (ESC) and the European Society of Anaesthesiology (ESA). Eur Heart J. 2014;35:2383-431.

8. Fleisher LA, Fleischmann KE, Auerbach AD, Barnason SA, Beckman JA, Bozkurt B, et al. 2014 ACC/AHA guideline on perioperative cardiovascular evaluation and management of patients undergoing noncardiac surgery: a report of the American College of Cardiology/American Heart Association Task Force on Practice Guidelines. J Am Coll Cardiol. 2014;64:e77-137.

9. Bettencourt N, Rocha J, Carvalho M, Leite D, Toschke AM, Melica B, et al. Multislice computed tomography in the exclusion of coronary artery disease in patients with presurgical valve disease. Circ Cardiovasc Imaging. 2009:2:306-13.

10. Jakamy R, Barthélémy O, Le Feuvre C, Berman E, Boutekadjirt R, Cluzel P, et al. Accuracy of multislice computed tomography in the preoperative assessment of coronary disease in patients scheduled for heart valve surgery. Arch Cardiovasc Dis. 2012;105:424-31
11. Russo V, Gostoli V, Lovato L, Montalti M, Marzocchi A, Gavelli G, et al. Clinical value of multidetector CT coronary angiography as a preoperative screening test before non-coronary cardiac surgery. Heart. 2007;93:1591-8.

12. Meijboom WB, Mollet NR, Van Mieghem CA, Kluin J, Weustink AC, Pugliese F, et al. Pre-operative computed tomography coronary angiography to detect significant coronary artery disease in patients referred for cardiac valve surgery. $J$ Am Coll Cardiol. 2006;48:1658-65.

13. Bellomo R, Ronco C, Kellum JA, Mehta RL, Palevsky P. Acute renal fail ure: definition, outcome measures, animal models, fluid therapy and information technology needs: the Second International Consensus Conference of the Acute Dialysis Quality Initiative (ADQI) Group. Crit Care. 2004 8:R204-12.

14. Hadamitzky M, Achenbach S, Al-Mallah M, Berman D, Budoff M, Cademartiri F, et al. Optimized prognostic score for coronary computed tomographic angiography: results from the CONFIRM registry (COronary CT Angiography EvaluatioN For Clinical Outcomes: an InteRnational Multicenter) registry. J Am Coll Cardiol. 2013;62:468-76.

15. Abbara S, Blanke P, Maroules CD, Cheezum M, Choi AD, Han BK, et al. SCCT guidelines for the performance and acquisition of coronary computed tomographic angiography: a report of the Society of Cardiovascular Computed Tomography Guidelines Committee: endorsed by the North American Society for Cardiovascular Imaging (NASCI). J Cardiovasc Comput Tomogr. 2016;10: 435-49.

16. Yang DH, Kang JW, Kim HK, Choe J, Baek S, Kim SH, et al. Association between C-reactive protein and type of coronary arterial plaque in asymptomatic patients: assessment with coronary CT angiography. Radiology. 2014;272: 665-73.

17. Kurth T, Walker AM, Glynn RJ, Chan KA, Gaziano JM, Berger K, et al. Results of multivariable logistic regression, propensity matching, propensity adjustment, and propensity-based weighting under conditions of nonuniform effect. Am J Epidemiol. 2006;163:262-70.

18. Normand ST, Landrum MB, Guadagnoli E, Ayanian JZ, Ryan TJ, Cleary PD, et al. Validating recommendations for coronary angiography following acute myocardial infarction in the elderly: a matched analysis using propensity scores J Clin Epidemiol. 2001;54:387-98.

19. Opolski MP, Staruch AD, Jakubczyk M, Min JK, Gransar H, Staruch M, et al. CT angiography for the detection of coronary artery stenoses in patients referred for cardiac valve surgery: systematic review and meta-analysis. JACC Cardiovasc Imaging. 2016;9:1059-70.

20. James MT, Samuel SM, Manning MA, Tonelli M, Ghali WA, Faris P, et al. Contrast-induced acute kidney injury and risk of adverse clinical outcomes after coronary angiography: a systematic review and meta-analysis. Circ Cardiovasc Interv. 2013:6:37-43.

21. Tavakol M, Ashraf S, Brener SJ. Risks and complications of coronary angiog raphy: a comprehensive review. Glob J Health Sci. 2012;4:65-93.

22. Moos SI, van Vemde DN, Stoker J, Bipat S. Contrast induced nephropathy in patients undergoing intravenous (IV) contrast enhanced computed tomography (CECT) and the relationship with risk factors: a meta-analysis. Eur J Radiol. 2013;82:e387-99.

Key Words: perioperative risk evaluation, coronary computed tomography angiography, valvular heart surgery 

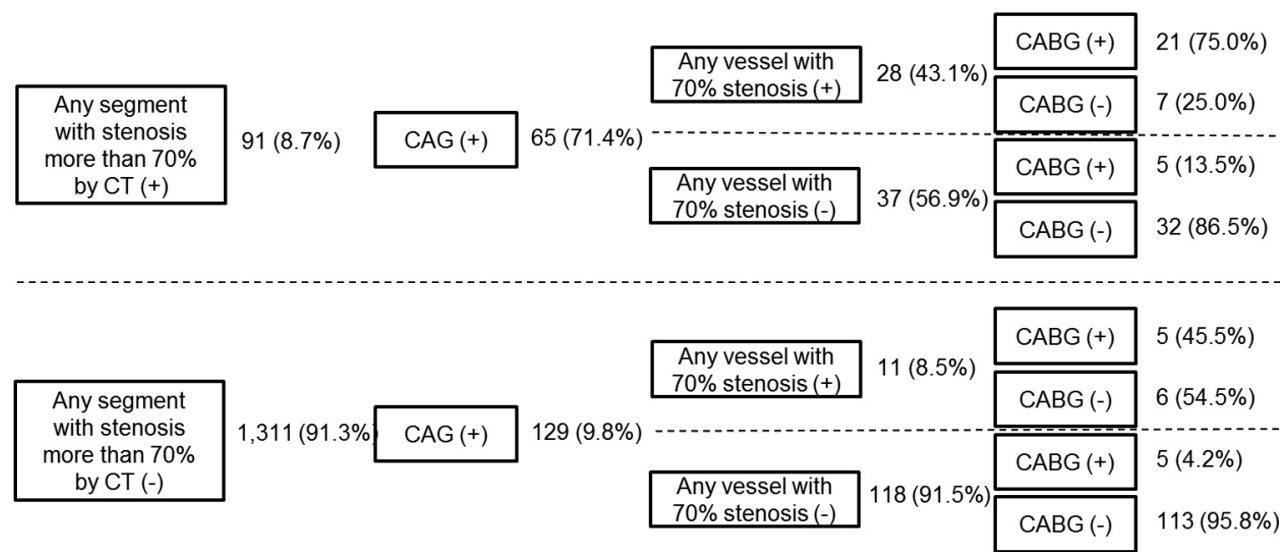

FIGURE E1. Clinical pathway of patients who initially underwent coronary computed tomography angiography for screening with a higher cutoff value of $70 \%$ stenosis $(\mathrm{n}=1420)$. $C T$, Computed tomography; $C A G$, coronary angiography; $C A B G$, coronary artery bypass grafting.
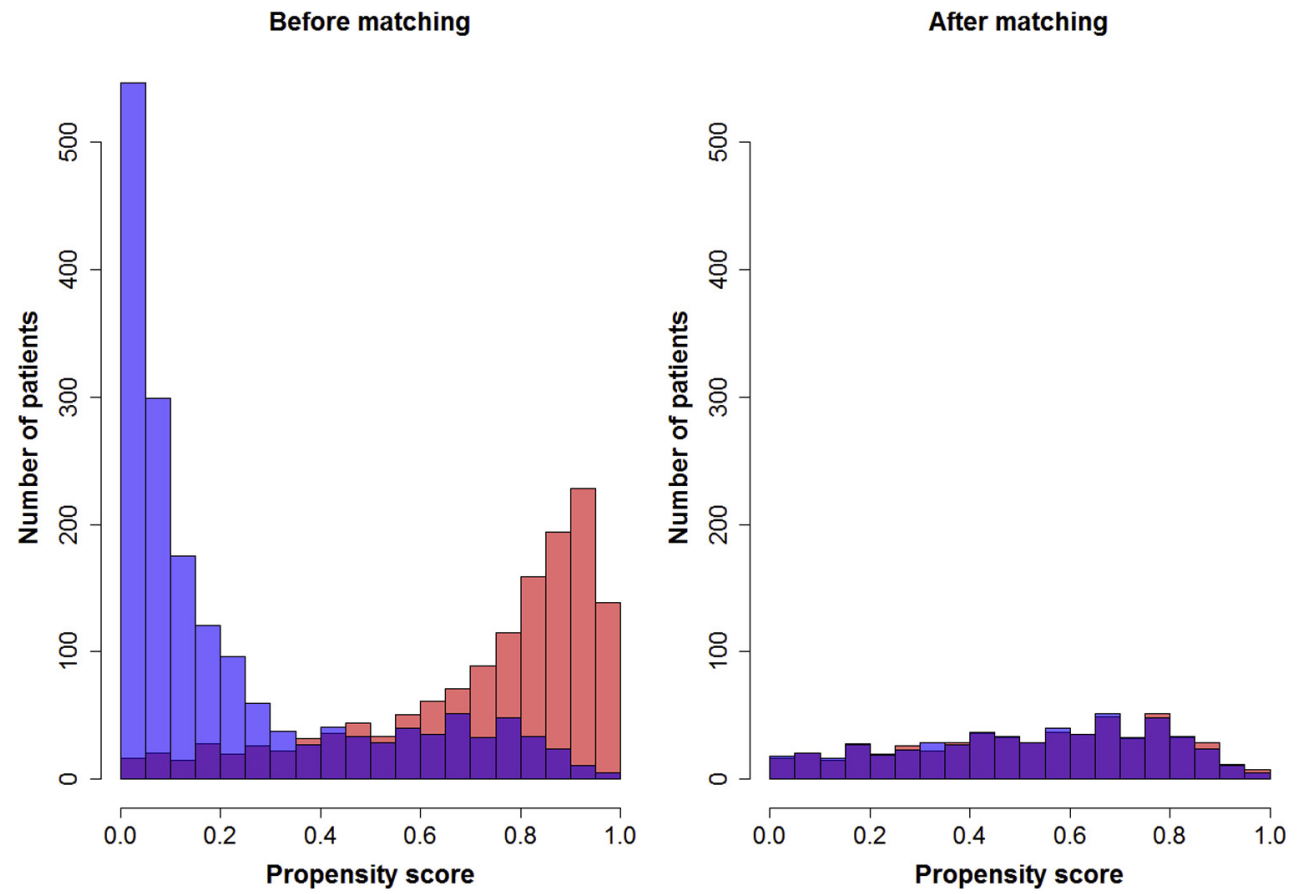

FIGURE E2. Distributions of propensity scores in conventional coronary angiography (blue) and coronary computed tomography angiography (red) groups before (left) and after (right) matching. 
TABLE E1. Imaging parameters, radiation dose, and diagnostic accuracy of coronary computed tomography angiography at Asan Medical Center

\begin{tabular}{llll}
\hline \multicolumn{1}{c}{ Parameter } & \multicolumn{1}{c}{ 16-slice CT } & First-generation dual-source CT & Second-generation dual-source CT \\
\hline CT model (vendor) & Sensation 16 (Siemens) & Definition (Siemens) & Definition Flash (Siemens) \\
\hline ECG gating mode & Retrospective & Retrospective or prospective & Retrospective or prospective \\
$\begin{array}{l}\text { Dose pulsing window in retrospective gating, } \\
\quad \text { R-R interval, \% }\end{array}$ & None & $20-70$ for semilunar valve & $20-70$ for semilunar valve \\
Body size adaptive radiation dose protocol & No & $30-80$ for mitral valve & $30-80$ for mitral valve \\
Tube voltage, $\mathrm{kVp}$ & 120 & Yes & Yes \\
Tube current-time product, $\mathrm{mAs}$ & 500 & 100 or 120 & 100 or 120 \\
Reported radiation dose, $\mathrm{mSv}$ & Not available & $240-400$ per rotation & $240-450$ per rotation \\
Reported diagnostic accuracy, \% & $80-81^{\mathrm{E} 3}$ & Not available & $8.7^{\mathrm{E} 2}$ \\
\hline
\end{tabular}

$C T$, Computed tomography; $E C G$, electrocardiography.

\section{E-References}

E1. Yang DH, Kang JW, Kim HK, Choe J, Baek S, Kim SH, et al. Association between C-reactive protein and type of coronary arterial plaque in asymptomatic patients: assessment with coronary CT angiography. Radiology. 2014;272:665-73.

E2. Han K, Yang DH, Shin SY, Kim N, Kang JW, Kim DH, et al. Subprosthetic pannus after aortic valve replacement surgery: cardiac CT findings and clinical features. Radiology. 2015;276:724-31.

E3. Choi JW, Seo JB, Do KH, Choi SI, Lee W, Ko SM, et al. Comparison of transaxial source images and 3-plane, thin-slab maximal intensity projection images for the diagnosis of coronary artery stenosis with using ECG-gated cardiac CT. Korean J Radiol. 2006;7:20-7.

E4. Yang DH, Kim YH, Roh JH, Kang JW, Ahn JM, Kweon J, et al. Diagnostic performance of on-site CT-derived fractional flow reserve versus CT perfusion. Eur Heart J Cardiovasc Imaging. 2017;18:432-40.

E5. Yang DH, Kim YH, Roh JH, Kang JW, Han D, Jung J, et al. Stress myocardial perfusion $\mathrm{CT}$ in patients suspected of having coronary artery disease: visual and quantitative analysis-validation by using fractional flow reserve. Radiology. $2015 ; 276: 715-23$
TABLE E2. Details of coronary lesions and bypass grafting in patients undergoing concomitant coronary bypass surgery

\begin{tabular}{lcc}
\hline \multicolumn{1}{c}{ Parameter } & $\mathbf{n}(\%)$ & $\mathbf{C A G}(\mathbf{n}=\mathbf{7 7})$, \\
$\mathbf{n}(\%)$
\end{tabular}

$C T$, Computed tomography; $C A G$, coronary angiography; $L A D$, left anterior descending artery; LITA, left internal thoracic artery; RITA, right internal thoracic artery; BITA, bilateral internal thoracic artery. 
TABLE E3. Causes of low cardiac output syndrome in the overall and propensity score-matched cohorts

\begin{tabular}{|c|c|c|c|c|c|c|}
\hline \multirow[b]{2}{*}{ Cause } & \multicolumn{3}{|c|}{ Overall cohort $(n=3150)$} & \multicolumn{3}{|c|}{ Propensity score-matched cohort $(n=1126)$} \\
\hline & $\begin{array}{c}\overline{\text { CT }(n=1402),} \\
n(\%)\end{array}$ & $\begin{array}{c}\text { CAG }(n=1748), \\
n(\%)\end{array}$ & $P$ value & $\begin{array}{c}\text { CT }(\mathbf{n}=563), \\
\text { n }(\%)\end{array}$ & $\begin{array}{c}\text { CAG }(\mathbf{n}=\mathbf{5 6 3}), \\
\mathbf{n}(\%)\end{array}$ & $P$ value \\
\hline PMI & $20(1.4)$ & $12(0.7)$ & .060 & $8(1.4)$ & $2(0.4)$ & .057 \\
\hline Cardiac dysfunction, PMI unrelated & $4(0.3)$ & $1(0.1)$ & .251 & $1(0.2)$ & 0 & $>.99$ \\
\hline Others* & $8(0.6)$ & $5(0.3)$ & .338 & $4(0.7)$ & $2(0.4)$ & .682 \\
\hline
\end{tabular}

$C T$, Computed tomography; $C A G$, coronary angiography; $P M I$, perioperative myocardial infarction, as defined by elevation of cardiac enzyme ( $>10$ times of reference value) associated with ECG changes (ST-T changes, new LBBB or Q waves). *Including bleeding, vasoplegic shock, and arrhythmia.

TABLE E4. Causes of early mortality

\begin{tabular}{|c|c|c|}
\hline Cause & $\begin{array}{c}\text { CT }(\mathbf{n}=23) \\
n(\%)\end{array}$ & $\begin{array}{c}\text { CAG }(n=23), \\
n(\%)\end{array}$ \\
\hline Low cardiac output syndrome & $12(52.2)$ & $8(34.8)$ \\
\hline Bleeding & $4(17.4)$ & $5(21.7)$ \\
\hline Stroke & $2(8.6)$ & $2(8.7)$ \\
\hline Multiorgan failure & $2(8.7)$ & $1(4.3)$ \\
\hline Prosthetic endocarditis & 0 & $1(4.3)$ \\
\hline Respiratory & $2(8.7)$ & $2(8.7)$ \\
\hline Sudden cardiac arrest & 0 & $1(4.3)$ \\
\hline Unknown* & $1(4.3)$ & $3(13.0)$ \\
\hline
\end{tabular}

$C T$, Computed tomography; $C A G$, coronary angiography. *Mortality cases after hospital discharge but within postoperative 30 days. 